\title{
Spontaneous rhythmic motor unit potentials in the carpal tunnel syndrome
}

\author{
F SPAANS \\ From the Departments of Clinical Neurophysiology, De Wever Hospital, Heerlen, and State University \\ of Limburg, Maastricht, The Netherlands
}

SUMMARY In 18 out of 164 consecutive patients with carpal tunnel syndrome ongoing spontaneous rhythmic motor unit potentials were found in the lateral thenar muscles. The frequency ranged from 3 to $9 \mathrm{~Hz}$ with an average of $6 \mathrm{~Hz}$. The spontaneous rhythmic motor unit potentials appeared to be doublets in many cases. Ischaemia usually caused a marked increase of spontaneous activity within 1 to 2 minutes. Directly after ischaemia the spontaneous rhythmic motor unit potentials subsided for some time. Variable periods of suppression also occurred after forceful muscle contraction and during repetitive supramaximal stimulation of the median nerve. In four patients where the median nerve had been blocked at the level of the elbow with lidocaine the spontaneous activity remained, indicating that the site of origin was distal, probably at the compression site at the carpus.

Concentric needle EMG (CNEMG) may reveal several forms of spontaneous activity. In normal conditions insertion activity and endplate potentials, may be encountered; any other spontaneous activity is considered to be pathological. Spontaneous muscle activity may have its origin in the muscle fibres themselves (myogenic spontaneous activity) or in the anterior horn cell or its axon (neurogenic spontaneous activity).

Fibrillation potentials, positive sharp waves, myotonic discharges, and bizarre high frequency discharges have a myogenic origin. They are potentials of single muscle fibres, isolated or grouped, with a rhythmic character.

Spontaneous neurogenic potentials are always motor unit potentials. When they are sporadic they are called "fasciculation potentials". Repetitive discharges of one motoneuron may occur in the form of "doublets": two action potentials of the same form and nearly the same amplitude, occurring consistently in the same relation to one another at an interval of 2.5-20 ms. ${ }^{1}$ They may occur spontaneously or during voluntary activation. Three or more of such potentials may form "triplets" and "multiplets" respectively. Irregular grouped or polyphasic spon-

Address for reprint requests: Dr F Spaans, Department of Clinical Neurophysiology, De Wever Hospital, Postbox 4446, 6401 CX Heerlen, The Netherlands.

Received 1 August 1981

Accepted 26 September 1981 taneous motor unit potentials are seen in hemifacial spasms. Spontaneous motor unit potentials may occur in central motor disorders in regular groups (tremor, clonus), completely irregular groups (for example chorea, myoclonus), and also as prolonged activity (spasticity). Finally, spontaneous motor unit potentials may occur in a strictly rhythmic ongoing way. It appears that these spontaneous rhythmic motor unit potentials may be found relatively often in the lateral thenar muscles of patients with the carpal tunnel syndrome. In the literature little attention has been paid to this remarkable activity. In this article a study of the characteristics of spontaneous rhythmic motor unit potentials has been reported.

\section{Subjects and methods}

In a period of 6 months 164 patients were seen in our department with a diagnosis of carpal tunnel syndrome based on clinical as well as on electrophysiological signs. Distributions of age and sex in this group are shown in fig 1. CNEMG of the abductor pollicis brevis and opponens pollicis (lateral thenar) muscles and motor and sensory conduction studies of the median and ulnar nerves were performed in all patients on both sides. Sensory conduction studies were done using the antidromic method: the main trunk of the nerve was stimulated supramaximally as in motor conduction studies, using surface electrodes. The sensory potentials were registered with ring electrodes $3 \mathrm{~cm}$ apart with the proximal electrode at the first interphalangeal joint. In the motor conduction studies a concentric needle electrode 


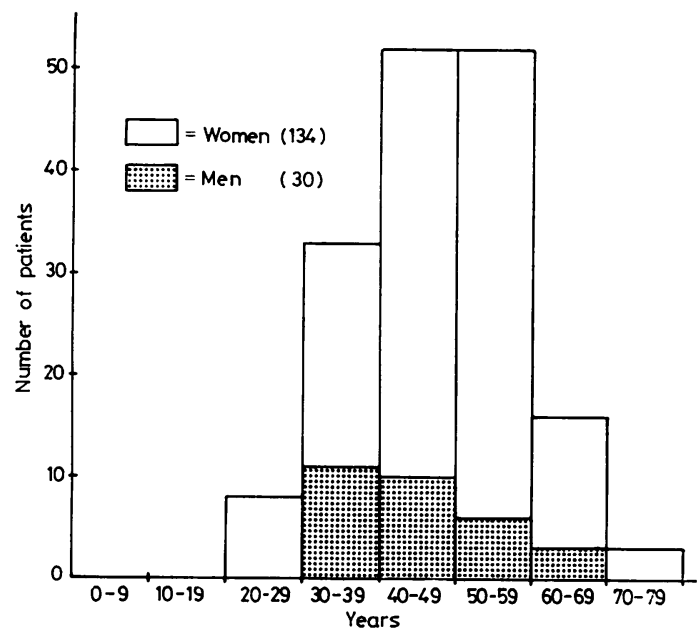

Fig 1 Distribution of age and sex in the group of 164 carpal tunnel syndrome patients.

in the abductor pollicis brevis muscle was used for registration. The distance between the needle and the stimulating cathode at the wrist was about $7 \mathrm{~cm}$. For supramaximal stimulation rectangular pulses of $0.2 \mathrm{~ms}$ duration and a voltage between 150 and $250 \mathrm{~V}$ were used. All studies were done with a skin temperature at the wrist between 32 and $34^{\circ} \mathrm{C}$.

The mildest carpal tunnel syndromes were diagnosed using the criteria put forward by Brenninkmeyer. ${ }^{2}$ Comparison of distal sensory latencies to the fourth finger on stimulation of the median and ulnar nerves at the wrist, appeared to be the most sensitive criterion. With equal distances between site of stimulation and proximal ring electrode a difference in latency time between the two nerves of $0.4 \mathrm{~ms}$ or more was considered to be pathological. If CNEMG of the lateral thenar muscles revealed spontaneous rhythmic motor unit potentials the effects of several manoeuvres were investigated in a number of patients: voluntary activation of the muscle, repetitive supramaximal stimulation of the median nerve, ischaemia by inflating a cuff around the upper arm, and blocking of conduction in the median nerve with $2 \%$ lidocaine. Additionally, SFEMG of the lateral thenar muscles was performed in three patients using a needle with an electrode surface of $25 \mu \mathrm{m}$.

\section{Results}

DISTAL MOTOR LATENCIES AND

SPONTANEOUS ACTIVITY

In 99 of the 164 patients carpal tunnel syndrome was diagnosed on both sides. In 66 of these cases the distal motor latency (DML) was increased most on the right side, in 28 cases most on the left side, whereas in two cases the same DML was found on both sides. In 47 patients only a right sided carpal tunnel syndrome was diagnosed, and in 18 patients a carpal tunnel syndrome was diagnosed only on the left side. Altogether the 164 patients had 263 carpal tunnel syndromes. Figure 2 shows a classification of the cases based on the DMLs of the left and right median nerves. Cases showing spontaneous muscle fibre potentials ( $=$ fibrillation potentials or positive sharp waves or both) and cases showing spontaneous rhythmic motor unit potentials are indicated in the columns. Spontaneous muscle fibre potentials were found in the lateral thenar muscles of 23 patients $(14 \%)$, nine times on the left side and 20 times on the right side. Spontaneous rhythmic motor unit potentials were found in the lateral thenar muscles of 18 patients, 11 times on the left side and 9 times on the right side (that is in $7.6 \%$ of the cases). Fasciculation potentials were seen in the lateral thenar muscles of four patients. In conclusion: distal motor latencies as well as spontaneous muscle fibre potentials dominated on the right side, but spontaneous rhythmic motor unit potentials were equally found on both sides, often in cases with minor conduction disturbances.

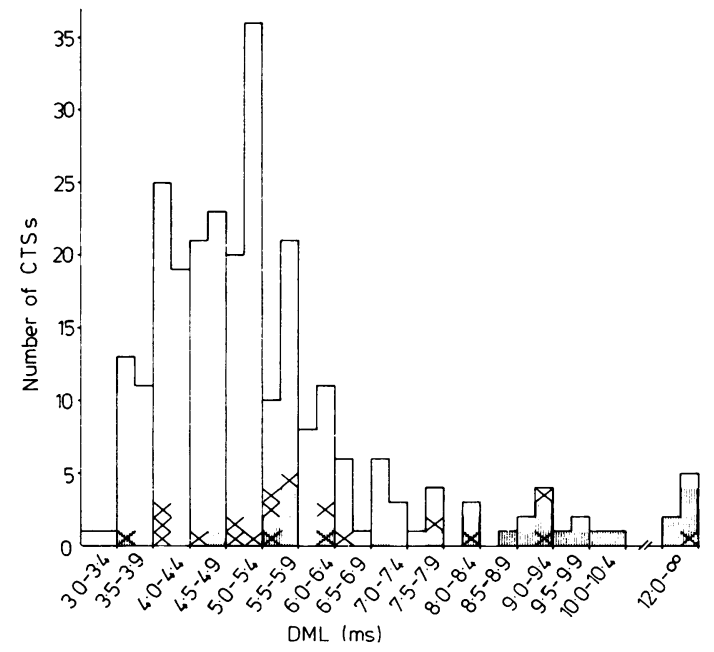

Fig 2 Distal motor latencies (DML) in 263 carpal tunnel syndrome patients (164 patients). The columns are divided in left and right halves, representing the left and right hands. $\times=$ spontaneous rhythmic motor unit potentials in the lateral thenar muscles. $|1 \mathrm{III}|=$ fibrillation potentials and/or positive sharp waves in the lateral thenar muscles (= spontaneous muscle fibre potentials).

APPEARANCE, FORM, AND FREQUENCY OF SPONTANEOUS RHYTHMIC MOTOR UNIT POTENTIALS

In the 20 thenar eminences where spontaneous rhythmic motor unit potentials were found there 
was usually only one such repetitive potential. If the active motor unit was rather distant from the recording surface of the needle electrode a very small potential was registered, which could easily be overlooked. The lateral thenar muscles were thoroughly investigated using two skin penetrations 5 to $10 \mathrm{~mm}$ apart in a line perpendicular on the fibre direction. If a spontaneous rhythmic motor unit potential was noticed the electrode was carefully displaced until the potential was registered with its highest amplitude. Frequently the spontaneously active motor unit was situated rather superficially, but contractions or rhythmic needle movements were not noticed. It was always impossible for the patient to suppress the rhythmic potential by efforts to reach further relaxation of the muscle or by activation of antagonistic muscles. Spontaneous rhythmic motor unit potentials appeared as single potentials (fig 3), doublets (fig 4), or triplets (fig 5). Sometimes they appeared to be very polyphasic (fig 6). Slight displacements of the needle electrode caused pronounced changes of the shape of the potentials. Usually the spontaneous rhythmic

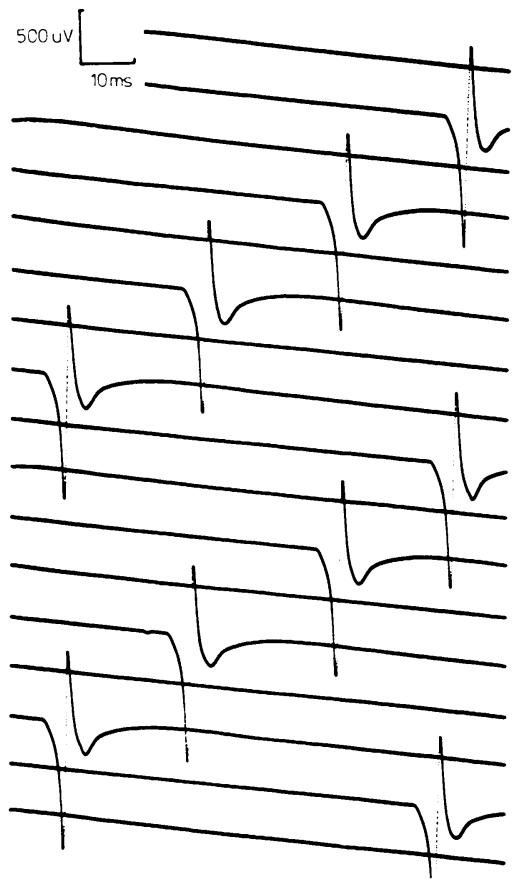

Fig 3 Spontaneous rhythmic motor unit potential in the left abductor pollicis brevis in a case of carpal tunnel syndrome (DML $4.6 \mathrm{~ms}$ ). Registration in "raster mode"; between the end of an upper trace and the start of the next lower trace a piece of about $3 \mathrm{~mm}$ is lost. The registration shows a strictly regular single motor unit potential with a frequency of $6 \mathrm{~Hz}$.

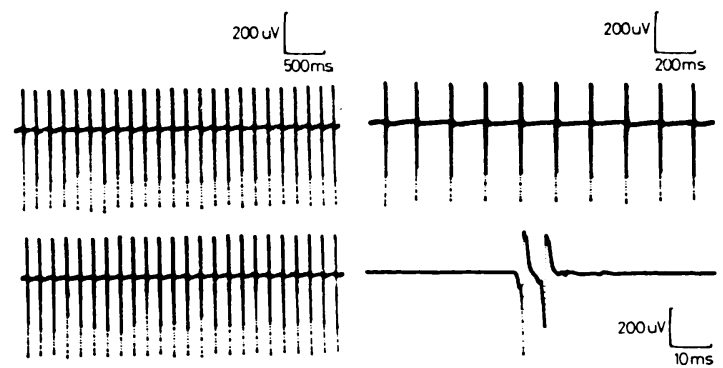

Fig 4 Spontaneous rhythmic motor unit potential in the abductor pollicis brevis in a case of carpal tunnel syndrome (DML 4.1 ms). Regular ongoing discharge consisting of doublets. Frequency $6 \frac{1}{2} \mathrm{~Hz}$.

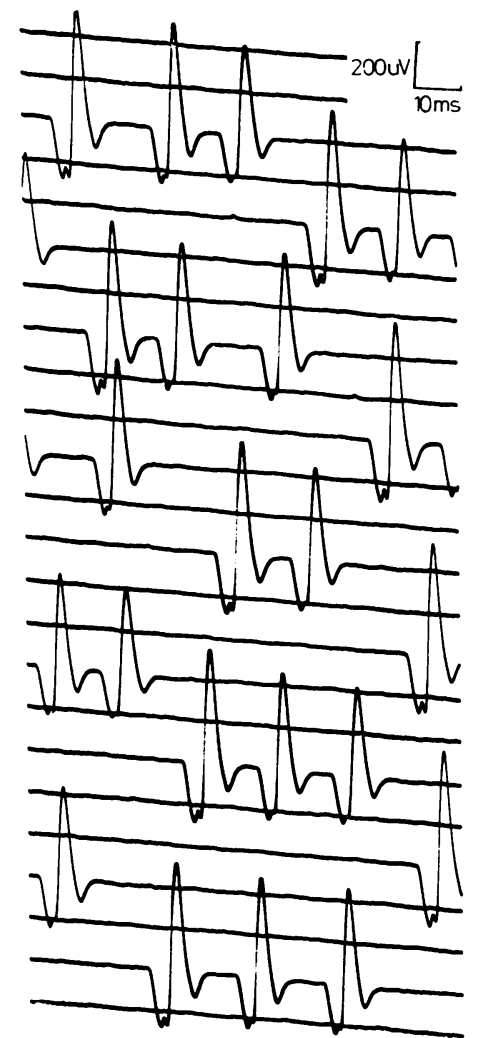

Fig 5 Spontaneous rhythmic motor unit potentials in the form of triplets $\left(4 \frac{1}{2} \mathrm{~Hz}\right)$ in the left abductor pollicis brevis (DML $5.5 \mathrm{~ms}$ ). Registration in "raster mode". There are rather pronounced interval variations between the components. The third component twice drops out.

motor unit potentials showed a strict rhythm (fig 4), but now and then a potential dropped out (fig 6) in a number of cases, and sometimes the spontaneous rhythmic motor unit potentials appeared in groups (fig 7). In several cases more than one spontaneous 


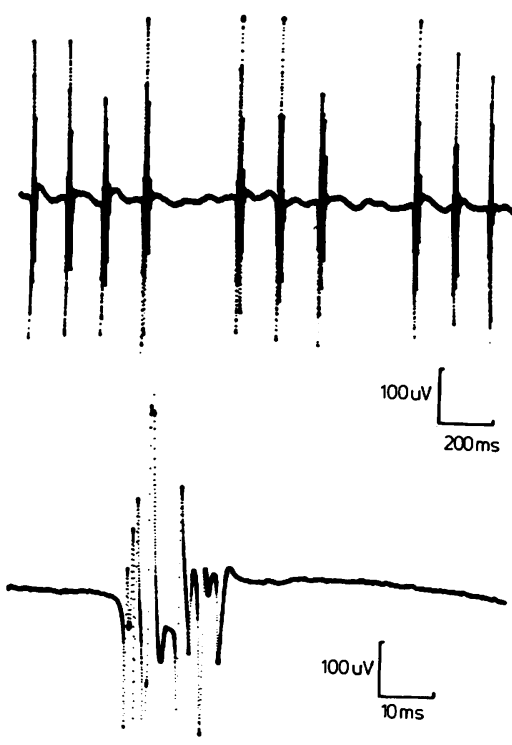

Fig 6 Strongly polyphasic spontaneous rhythmic motor unit potential with occasional dropouts in the opponens pollicis in a case of carpal tunnel syndrome (DML 6.7 ms).
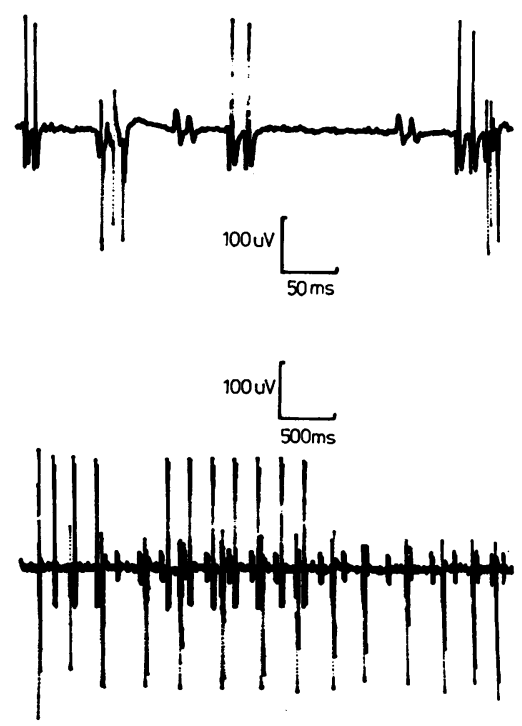

Fig 7 Three spontaneous doublets registered in the lateral thenar eminence in a case of carpal tunnel

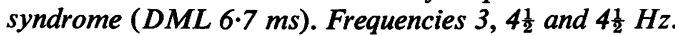
The doublet with the highest upward deflection shows a periodic dropout. rhythmic motor unit potential was recorded from one position of the needle electrode (fig 7). The frequency of the spontaneous rhythmic motor unit potentials ranged from 3 to $9 \mathrm{~Hz}$ with an average of $6 \mathrm{~Hz}$. Dropout of potentials and especially combinations of several spontaneous rhythmic motor unit potentials (fig 7) sometimes produced a musical or even swinging sound on the EMG loudspeaker. How long the rhythmic potentials may continue is unknown. In one patient seen again after 9 days the potential could no longer be shown. In another patient the potential was still present after 3 days. Only in one case was the spontaneous rhythmic motor unit potential lost during the investigation.

\section{SINGLE FIBRE EMG}

In three patients the spontaneous rhythmic motor unit potentials were investigated with a single fibre electrode. The electrode was carefully positioned until the small leading off surface was close to a number of active muscle fibres. Figures 8 and 9

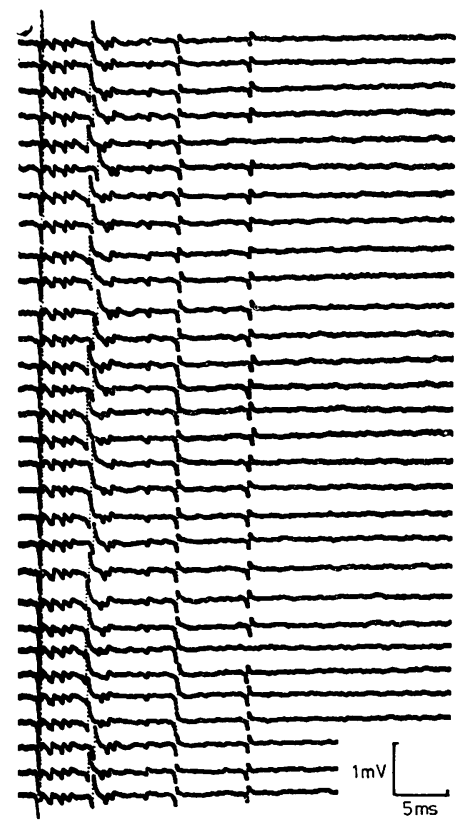

Fig 8 SFEMG of the same spontaneous potential as in fig 4. Successive discharges filmed from top to bottom. The first potential triggers the sweep of the oscilloscope. The complex is shifted to the right by using a delay line. There is a pronounced "jitter" of the second component of the doublet, in which the 3 small late potential do not participate, indicating that they belong to the first component. The last potential drops out two times. In the second component of the doublet the occasional dropout of the second $S F$ potential is already visible (see also fig 9). 
(a)

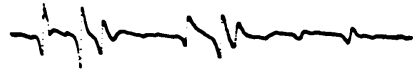

(b)

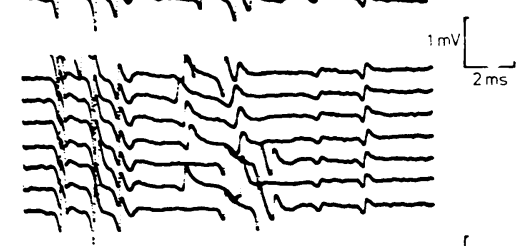

(c)

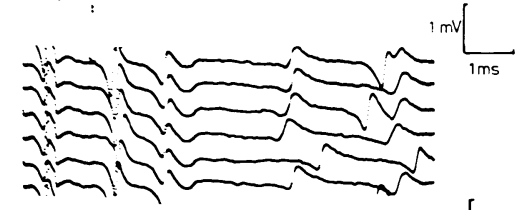

(d)

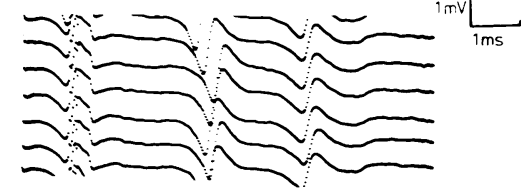

(e)

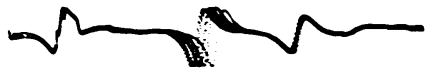

Fig 9 SFEMG of the same spontaneous doublet as in fig 8. There is a slight change in needle position compared with that figure. Because of the shorter time base the last potential of the complex is lost already in $9 a$ and $9 b$. (a) "Single shot" registration for a better look at the individual SF potentials. The 3 potentials of the second component are lower and broader than the corresponding potentials of the first component. (bc) To show large "jitter" of the second component of the doublet with large real jitter and often blocking of the second SF potential.

(de) Increased real jitter of the second SF potential of the first component. In (e) 4 traces have been superimposed. The negative going slope of the first potential is deformated by a trigger marker.

show single fibre registrations of the doublet of which the CNEMG registration is shown in figure 4. There appears to be a pronounced interval variation between the two components of the doublet. This is not a "jitter" in the usual sense. The second complex contains single fibre potentials from the same muscle fibres as the first complex. It is clear from the figure that the potentials in the second complex are lower and broader than those of the first complex (see discussion). The two late potentials seem to belong to the first complex because they do not show the same variance in interval as the second complex does. Twice a dropout of the latest potential may be notified. In fig 9 it is shown that there are also real pathological jitters within the complexes.
EFFECTS OF VOLUNTARY ACTIVATION OF

THE LATERAL THENAR MUSCLES

In six patients slight contraction of the lateral thenar muscles caused an increase in frequency of the spontaneous rhythmic motor unit potentials. In most cases there was no distinct effect, or the spontaneous rhythmic motor unit potential was lost between the voluntary potentials. Maximal contraction was often, but not always, followed by a variable period of suppression of spontaneous rhythmic motor unit potentials (fig 10).

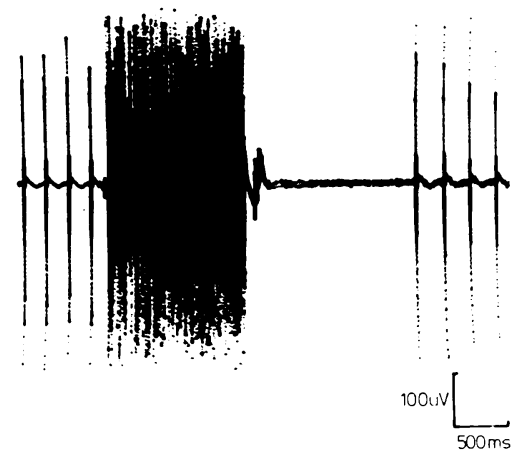

Fig 10 Example of suppression of spontaneous motor unit activity following short-lasting contraction of the abductor pollicis brevis muscle.

EFFECTS OF SUPRAMAXIMAL ELECTRICAL STIMULATION OF THE MEDIAN NERVE In a number of cases supramaximal stimulation of the median nerve at the wrist caused a depression of the spontaneous rhythmic motor unit potentials. There was no clear relationship between the moment of stimulation and the occurrence or duration of the pauses in the spontaneous activity (fig 11). Whether or not the site of spontaneous depolarisation of the nerve fibre was passed by the electrically evoked impulse could not be decided. In moderate and severe cases of carpal tunnel syndrome the evoked potential, recorded with a concentric needle electrode from the lateral thenar muscles, was often polyphasic with an increased duration. In a number of cases, with or without spontaneous rhythmic motor unit potentials, the evoked potential contained late components comparable with those shown in the SF registration of fig 8 . Figure 12 shows that such a late component can just as well form a part of the $M$ response as of the $F$ response. Obviously such late components are a result of slow conduction in a sprout of a motor axon. The interval with the main component was more than $100 \mathrm{~ms}$ in three cases in this series. In two patients without spontaneous rhythmic motor unit potentials 

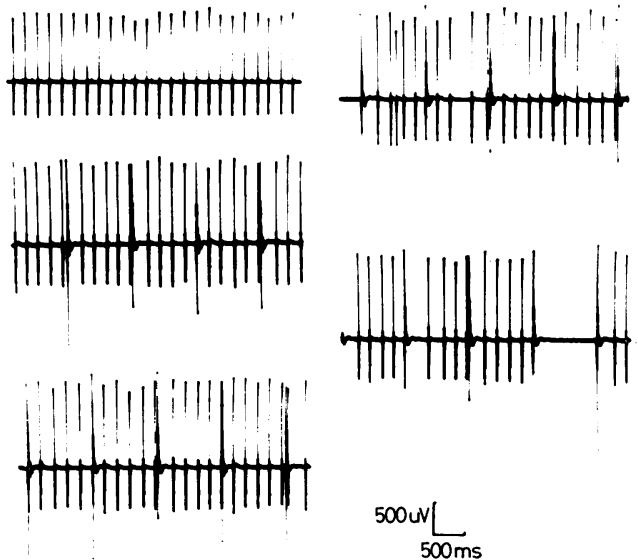

Fig 11 Effect of supramaximal $1 \mathrm{~Hz}$ stimulation of the median nerve at the wrist on strictly regular spontaneous rhythmic motor unit potentials in the abductor pollicis brevis. Often, but not always, a small delay of the following spontaneous rhythmic motor unit potential is caused and one time a longer lasting suppression occurs. The highest potentials are the evoked muscle potentials.

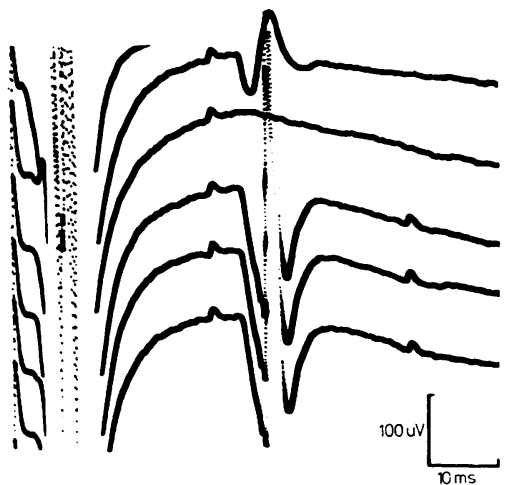

Fig $12 M$ and $F$ responses from the abductor pollicis brevis evoked by supramaximal stimulation of the median nerve at the wrist in a mild case of carpal tunnel syndrome (DML $3.5 \mathrm{~ms}$ ). The $M$ response always contains a small late component, which is also present as a part of the $F$ response in the last three traces. In the upper trace the motoneuron with the late component is apparently not activated by the antidromic volley, and in the second trace there is no $F$ response at all.

a repetitive discharge occurred with a latency somewhat longer than that of the $F$ response and consisting of a changing number of one to eight monomorphic potentials (fig 13).
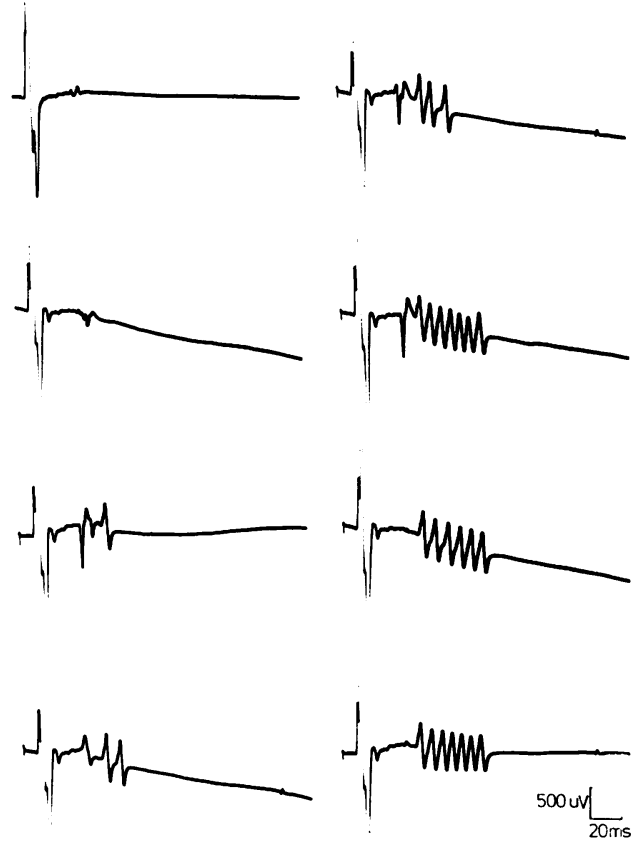

Fig 13 Potentials recorded with a concentric needle electrode from the right abductor pollicis brevis muscle in a case of carpal tunnel syndrome (DML:8.0 ms) evoked by supramaximal stimulation of the median nerve at the wrist. With a latency somewhat longer then that of the $F$ response there often occurred a discharge consisting of 1 to 8 monomorphic potentials. The traces are selected from a larger number. In this muscle also a spontaneous rhythmic motor unit potential was found.

EFFECTS OF ISCHAEMIA

In nine patients with spontaneous rhythmic motor unit potentials in the lateral thenar muscles ischaemia was caused by inflating a cuff around the arm. In all cases the amount of spontaneous rhythmic motor unit potentials increased within one to two minutes, usually with an increase in frequency, and sometimes moreover by the appearance of additional spontaneous rhythmic motor unit potentials (fig 14). Sometimes the increase was very pronounced. Directly after the cuff was released the spontaneous rhythmic motor unit potentials usually suddenly diminished or completely disappeared for up about 20 seconds and then gradually increased to regain the original frequency (fig 14). In four patients with moderate conduction disturbances of the median nerve at the wrist, but without spontaneous rhythmic motor unit potentials, ischaemia lasting 3 to 5 minutes failed to produce spontaneous rhythmic motor unit potentials. 

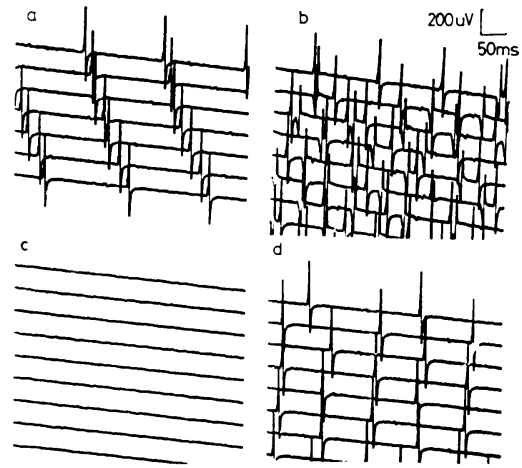

Fig 14 Effect of ischaemia on spontaneous motor unit activity in the abductor pollicis brevis muscle in a moderate case of carpal tunnel syndrome (DML:5.9 ms). (a) Before inflating the cuff around the arm. (b) Cuff inflated around the arm above systolic pressure for about 70 seconds: increased frequency of the original potential and developing of a new spontaneous rhythmic motor unit potential (which begins with a downward deflection). (c) A few seconds after release of the cuff, (d) Ten seconds later. Amplification and time base in all pictures as indicated in $b$.

\section{EFFECTS OF BLOCKING OF THE MEDIAN}

NER VE

The effect of blocking of the median nerve with $5 \mathrm{ml}$ $2 \%$ lidocaine was originally studied with injection at the wrist in two patients. In one patient the potentials remained and in the other they disappeared. It seemed however possible that the anaesthetic could have reached the site of nerve compression so that the eventual generator of pathological nerve activity might have been influenced. In order to avoid this problem in four other patients with spontaneous rhythmic motor unit potentials the median nerve was blocked at the level of the elbow. The existence of a total block in the motor fibres was ascertained by efforts of voluntary activation and by supramaximal stimulation distal and proximal to the injection site. In all these four cases the spontaneous rhythmic motor unit potentials remained with their original frequency. Total motor blockade was usually achieved after 20 to 30 minutes. The optimal site for injection was found by using the injection needle as a stimulating cathode.

\section{Discussion}

It is well known that the carpal tunnel syndrome occurs more frequently in women than in men. Also the preponderance of the age groups between 40 and 60 years, as can be seen in fig 1 , is a well known fact. The men in our group were all older than 30 years, but there was no further age relationship. The only information that could be found about this matter in the literature ${ }^{3}$ showed the same situation.

Whereas the diagnosis of mild cases of carpal tunnel syndrome in the present series was based on sensory studies, values of DMLs have been used in fig 2 to create sub-groups. A reason for this was that in more severe cases no antidromic sensory potential could be recorded. It was also an advantage that in this way motor features could be correlated with each other: DMLs on one side and spontaneous muscle fibre and motor unit potentials on the other side. Usually DMLs of the median nerve up to $4.5 \mathrm{~ms}$ are considered to be in the normal range. In this series the diagnosis of carpal tunnel syndrome could be confirmed in 69 hands with a DML of less than $4.5 \mathrm{~ms}$. This demonstrates the superiority of sensory latency studies in these patients; in my experience comparison of the distal sensory latencies of the median and ulnar nerves to the fourth finger as proposed by Brenninkmeyer ${ }^{2}$ (see Subjects and Methods) is the most sensitive criterion.

From fig 2 it is evident that the disturbances dominate on the right side not only with regard to the total number of cases but also to the number of cases with fibrillation potentials or positive sharp waves or both. Spontaneous rhythmic motor unit potentials however were equally found on both sides and often in cases with minor conduction disturbances.

In the majority of the 22 cases with a DML of $8 \mathrm{~ms}$ of more spontaneous muscle fibre potentials were found in the lateral thenar muscles. They were absent only in four cases. In one of these there was no activity at all in the lateral part of the thenar and on stimulation of the median nerve to motor response could be obtained $(\mathrm{DML}=\infty)$.

Out of this group of 22 with severe carpal tunnel syndrome spontaneous rhythmic motor unit potentials occurred in four cases, three times in combination with spontaneous muscle fibre potentials. In the cases with shorter DMLs there was less spontaneous muscle fibre activity, but spontaneous rhythmic motor unit potentials were found relatively more frequently. In a number of severe cases spontaneous rhythmic motor unit potentials were found on the less affected opposite side, which was relatively often the left side.

With respect to the nature of the spontaneous rhythmic motor unit potentials a number of questions need to be answered, the first of which is: "Are spontaneous rhythmic motor unit potentials really motor unit potentials? Are they generated in the motoneuron or might they be myogenic?' It has been shown in this study that the ongoing spontaneous rhythmic discharges always consist of more 
than one muscle fibre potential. This is however also the case with high frequency bizarre discharges as well as with the low frequency bizarre discharges, ${ }^{4}$ which are considered to be myogenic. With the low frequency bizarre discharges the spontaneous rhythmic motor unit potentials have the frequency range in common. The bizarre discharges however show a very low jitter of their components, which is explained by the circumstance that no neuromuscular transmission plays a part; the series of potentials are thought to be a result of ephaptic transmission between muscle fibres. ${ }^{5}$ Apart from the interval variations in cases of spontaneous doublets, the single fibre potentials belonging to one spontaneous rhythmic motor unit potential often show a pathological increased jitter (fig 9 de), which points to a neurogenic origin. Thus the spontaneous rhythmic motor unit potentials may be generated in the anterior horn cells or somewhere in the axon. The persistence of the spontaneous rhythmic motor unit potentials after total blockade of motor conduction at the level of the elbow proves that the site of origin must be distal to the elbow, presumably at the site of nerve compression at the wrist. Doublets, spontaneous or by activation, are to be considered as a repetitive firing of a motoneuron by a decreased stability of a part of its membrane.

The interval between the two depolarisations is often shorter than the relative refractory period of a number of muscle fibres. This results in lower and broader single fibre potentials in the second component of the doublet as compared with the corresponding potentials of the first component (see fig $9 \mathrm{abc}$ ). From fig 9 it is also evident that in the second component of the doublet the (real) jitter is much more pronounced that in the first component. Moreover the second SF potential is repeatedly absent in the second component, apparently because of blocking of the neuromuscular transmission. It is probable that a reduced safety factor in a recently formed endplate is responsible for this phenomenon. ${ }^{5}$ From fig 8 it is evident that the last three SF potentials belong to the first component of the doublet because they do not follow the large "jitter" of the second component. We cannot however find them back in the second component, which means that the interval between the two components at any time is shorter than the absolute refractory period of the muscle fibres in question or of their neuromuscular junctions. The last SF potential shows a failure of its neuromuscular transmission by two blockings in the first component. Together with the lower and broader SF potentials in the second component the increased jitter and the blockings will be responsible for the lower amplitude and slight shape differences of the second component of doublets as recorded by CNEMG.

A further important question concerns the mechanism which underlines the spontaneous rhythmic motor unit potentials. It is well known that nerve compression may lead to paraesthesia. That means that at the site of compression nerve impulses are generated in sensory neurons. For that reason it seems likely that also in motoneurons at a compression site nerve impulses would be generated, resulting in a contraction of the respective motor units. This is however rarely seen. We know of spontaneous motor unit potentials in the form of fasciculation potentials, usually due to anterior horn cell diseases and only rarely to root lesions or more distal nerve lesions. Fasciculation potentials are action potentials with the dimensions of motor unit potentials of either normal or abnormal size and form, which occur spontaneously and sporadically. ${ }^{1}$ In this series of carpal tissue syndrome patients fasciculation potentials were found in the lateral thenar muscles of four hands. Much more striking was the occurrence of spontaneous rhythmic motor unit potentials in $7.6 \%$ of the cases. This kind of activity has rarely and only superficially been reported in the literature. ${ }^{6} 7$ Exceptionally, it may also be found in other nerve compression syndromes, for example in radicular lesions. Recently I saw a woman with a brachial plexus invaded by a malignant tumour, who showed very vivid spontaneous rhythmic motor unit potentials in several muscles of the hand and fore-arm combined with fast small spontaneous movements. In carpal tunnel syndrome patients I have never observed spontaeous movements in combination with spontaneous rhythmic motor unit potentials, although the active motor unit sometimes had a rather superficial position. Sindermann et $a l^{8}$ describe the occurrence of "repetitive fasciculations" in one patient with an old poliomyelitis and in two healthy subjects. The discharges shown closely resemble the spontaneous rhythmic motor unit potentials in our patients. The authors supposed that the potentials were generated in or near the spinal cord: blocking of the radial nerve in one of the subjects at the level of the axilla caused the disappearance of the spontaneous potentials in the triceps brachii. In our four cases where the median nerve was blocked at the level of the elbow, the spontaneous rhythmic motor unit potentials persisted, which proves that the spontaneous activity was generated more distally. In three of the four cases the spontaneous rhythmic motor unit potentials consisted of doublets. The fact that the second component of the doublets remained after blocking of the nerve shows that the site of origin of this component was also distal 
(probably the site of compression) and could not for example be a result of triggering of the anterior horn cell by the first component.

According to Kugelberg ${ }^{9}$ the fact that motor excitation phenomena arising in nerve fibres occur so rarely under pathological conditions, is due to a better accommodation in the motor nerve fibres as compared with the sensory fibres. This means that in motor nerve fibres ongoning stimulation would result in a stronger increase of the threshold value than in sensory fibres. The accommodation of nerve fibres may be influenced in several ways. Apparently local compression of a nerve may cause a local breakdown of this capacity of the nerve to defenc, it might be said, itself against depolarisation, which results in spontaneous discharges. Kugelberg showed in experimental animals that ischaemia lowers the rheobase even after a few seconds. He also investigated healthy volunteers by inflating a pneumatic cuff around the arm above the systolic pressure and recording motor nerve activity indirectly from the first interosseus muscle with a concentric needle electrode. Paraesthesiae started after about one minute, reaching a maximum after two to five minutes, but motor activity was only obtained in isolated cases during ischaemia, and afterwards only if the pneumatic cuff had been kept on for a long time (about half an hour). Apparently the accommodation of motor fibres is less influenced than that of sensory fibres by ischaemia.

Kugelberg ${ }^{9}$ found that motor discharges during ischaemia could be improved by getting the experimental subject to hyperventilate for a few minutes before the cuff was placed around the arm. I have not used hyperventilation in the investigation of carpal tunnel syndrome patients. The effect of short lasting ischaemia on the already present spontaneous activity was evident (fig 4). Ischaemia for longer than five minutes was not used, not even in a number of patients where I sought for spontaneous rhythmic motor unit potentials that were absent without ischaemia. Continued ischaemia is rather unpleasant for the patient and it may also bring about spontaneous motor unit potentials in healthy persons. Combining ischaemia and hyperventilation Kugelberg found spontaneous discharges of 7 to 16 $\mathrm{Hz}$ "with a machine-like regularity" in those cases who developed a Trousseau phenomenon during the period of ischaemia. This frequency is rather close to what I found in the carpal tunnel syndrome cases $(3-9 \mathrm{~Hz})$. Adrian 10 recorded prolonged discharges from the injured end of a mammalian nerves. He found that three different kinds of discharges may occur. One consisted of a regular continuous series of impulses with a frequency over $150 \mathrm{~Hz}$, which could pass into a second type: irregular pulses with a lower frequency. The third type was characterised by groups of discharges, each consisting of impulses with high frequency, but each individual group recurring with a low frequency, 5 to $20 \mathrm{~Hz}$. Our spontaneous rhythmic motor unit potentials have a frequency which approximates to that of this last type. Adrian, however, found spontaneous discharges only in small sensory nerve fibres. Wall and co-workers ${ }^{11}$ could not reproduce Adrian's results. Prolonged injury discharges could only be produced by them if the cut end of a nerve was allowed to dehydrate or by severely reducing the calcium level of the damaged axons.

It is questionable to what extent the spontaneous activity in cases of carpal tunnel syndrome may be compared with that in the studies mentioned above. However, the present investigations also show three possible cycles of periodicity of the spontaneous activity generated in the so-called "parabiotic" part $^{6}{ }^{7}$ of the nerve fibre. The highest frequency is found in the components of spontaneous doublets and triplets (figs 4, 5, and 7) namely about $100 \mathrm{~Hz}$. These potentials are not strictly regular but show rather modest interval variations. The second category is formed by the spontaneous rhythmic motor unit potentials (single motor unit potential, doublets, or triplets), which are strictly regular with a frequency of 3 to $9 \mathrm{~Hz}$, usually about $6 \mathrm{~Hz}$. The third possible category consists of rather irregular periods of presence and absence of groups of spontaneous rhythmic motor unit potentials as can be seen in fig 7. Apparently these three rhythms are to be considered as expressions of an autorhythmic mechanism of the nerve fibre ${ }^{9}$ triggered by a pathologic stimulus. The fundamental aspects of this have, to my knowledge, not been elucidated so far.

In some cases the frequency of the spontaneous motor unit potentials increased during slight voluntary activation. The lack of effect in other cases is not necessarily due to impulse blocking at the parabiotic site of the nerve fibre. The spontaneously active motor unit may be a high threshold unit and therefore not be recruited during slight or moderate muscle contraction. Impulses elicited elsewhere in the nerve fibre may be blocked by any antidromic impulses from the parabiotic site. The question whether, additionally, nerve impulses can be blocked at the parabiotic site itself could not be answered, even from the results of supramaximal stimulation of the median nerve.

Often descending nerve impulses were able to suppress the spontaneous activity as has been shown by application of forceful voluntary activation and repetitive supramaximal nerve stimulation. Nerve stimulation could however also give rise to repetitive 
discharges (fig 13), possibly as an expression of activation of the parabiotic part of the nerve fibre. Such repetitive discharges in the carpal tunnel syndrome have been described by Simpson ${ }^{12}$ in 1956 , but in more recent literature this phenomenon is hardly mentioned.

In conclusion it may be stated that the carpal tunnel syndrome has to be considered not only as a frequent and rather trivial neurological problem, but at the same time as a laboratory model, given to us by nature, to study effects of long-lasting local compression of human nerve fibres. The aim of this article has been to focus attention on the occurrence and the features of ongoing spontaneous rhythmic motor unit potentials in the lateral thenar muscles of a number of patients with this syndrome, and on the challenge that these potentials provide for the understanding of nerve fibre pathophysiology.

\section{References}

${ }^{1}$ Simpson JA. Terminology of electromyography. Electroencephalogr Clin Neurophysiol 1969;26:224-6.

${ }^{2}$ Brenninkmeyer $\mathbf{R}$. The carpal tunnel syndrome and the antidromic sensory latencies to the first and the fourth finger. Acta Neurol Scand 1979; suppl. 73 60:119.

${ }^{3}$ Benini A. Das Karpaltunnelsyndrom und die übrigen Kompressions-syndrome des Nervus medianus. Thieme Stuttgart 1975. 92.

${ }^{4}$ Stoehr M. Low frequency bizarre discharges. Electromyogr Clin Neurophysiol 1978;18:147-56.

${ }^{5}$ Stålberg E, Trontelj JV. Single fibre electromyography. Mirvalle Press, Surrey 1979.

${ }^{6}$ Esslen E, Magun R. Elektromyographie, Grundlagen und klinische Anwendung. Fortschr Neurol Psychiat 1958;26:153-99.

7 Ruprecht EO. Befunde bei Neuropathien. In: Hopf HC, Struppler A (Eds). Elektromyographie. Lehrbuch und Atlas. Thieme Stuttgart 1974, 37-65.

${ }^{8}$ Sindermann F, Conrad B, Jacobi HM, Prochazka VJ. Unusual properties of repetitive fasciculations. Electroencephalogr Clin Neurophysiol 1973;35:173-9.

${ }^{9}$ Kugelberg E. "Injury activity" and "trigger zones" in human nerves. Brain 1946;69:310-24.

${ }^{10}$ Adrian ED. The effects of injury on mammalian nerve fibres. Proc Roy Soc B 1930;106:596-618.

11 Wall PD, Waxman S, Basbaum AI. Ongoing activity in peripheral nerve: injury discharge. Exp Neurol 1974;45:576-89.

12 Simpson JA. Electrical signs in the diagnosis of carpal tunnel and related syndromes. $J$ Neurol Neurosurg Psychiatry 1956;19:275-80. 